\title{
Active Contour Model Based on Nonlocal Means Method For Color Texture Segmentation
}

\author{
Jingge $\mathrm{Lu}^{1, \mathrm{a}}$, Guodong Wang ${ }^{2, \mathrm{~b}}$, Zhenkuan $\mathrm{Pan}^{3, \mathrm{c}}$ \\ ${ }^{1}$ College of Information Engineering, Qingdao University, Qingdao, 266071, China \\ ${ }^{2}$ College of Information Engineering, Qingdao University, Qingdao, 266071, China \\ ${ }^{3}$ College of Information Engineering, Qingdao University, Qingdao, 266071, China \\ aemail: cgjingge730@163.com, bemail:allen_wgd@163.com, cemail: zkpan@126.com
}

Keywords: Color texture segmentation; Active contour model; Nonlocal Tikhonov regularization; Image slice similarity; Split-Bregman algorithm

\begin{abstract}
Color texture segmentation is a very important subject in the fields of computer vision. In order to segment the color textures, a new method based on active contour model with nonlocal Tikhonov regularization is proposed. In detail, another smoothness item which is the Tikhonov regularization is added in the traditional active contour model. Meanwhile the nonlocal operator which is based on the image slice similarity is joined in the smoothness item. What's more, in order to improve the computation efficiency, this paper designs the Split-Bregman algorithm. At last, our performance is demonstrated by segmenting many complex and real texture images.
\end{abstract}

\section{Introduction}

Color image segmentation is useful in many applications. It is easy to distinguish interest regions of the general image by the segmentation results. But it is far from being an effortless task for color texture image segmentation. Due to the difficult nature of the problem, there are few automatic algorithms that can work well on a large variety of texture data. What's more, it is difficult to pick out the boundary between two textures because there is no sharp difference between them.

Recent years a lot of analogous researches have done. For example, Lui [1]use sparse texture model to detect the boundary of textures. The method integrated into the multi-function hidden markov model to detect the edges of the texture image. But it is very sensitive to initial position. Facts proved that when the textures' edge cannot represent regions of interest and have blurry contours, edge-based segmentation produce unsatisfactory result [2]. Meanwhile some methods also need to detect image's edge. The edge detection relies on the gradient of the image in some active contour models [3][4] of segmentation so that unclear boundaries is produced between two textures. To deal with the problem of edge detection, a method which is referenced from literature [5] combines image decomposition model and active contour model. But the result of this method is not very good which the contour line cannot be fully fit with the image's edge. What's more, image diffusion is appeared. A new approach called JSEG is proposed for color-texture segmentation in the reference [6]. However, there are two factors which will affect the segmentation results. Firstly, it is difficult to select a good color appropriate parameter when facing a strange image. Then there are different shades which will cause over segmentation because of the illumination or colors smooth transition.

The methods described above have limitations in different aspects more or less. To overcome image diffusion and edge detection and time consuming, the active contour model with nonlocal Tikhonov regularization is proposed. Because the images with a large of texture data cannot be segmented well only according to the pixel values, so the nonlocal operator is joined in the new smoothness item. The new smoothness item with nonlocal operator cannot be equal to the simple gradient item. The nonlocal means method is based on the image slice similarity rather than the pixel values. What's more, the final contour line can be close to the object edge. It avoids the image diffusion and the interference of the texture features. The segmentation results are satisfactory for the complicate images which have many texture characteristics. What's more, the Split-Bregman 
algorithm improves the computation efficiency which can save time.

The outline of the paper is as follows. The next section introduces the model for color textures segmentation. Then we describe the traditional active contour model for color image segmentation. At last, based on the nonlocal means method and the traditional active contour model, the model for color textures segmentation is proposed. What's more, we design the Split-Bregman algorithm [7]. At last, we make some numerical experiments. Because the conventional Mumford-shah model can't be use for the texture segmentation, so we make comparison with some models which are typical methods to use to segment color texture images.

\section{The model for color textures segmentations}

The variational formula of nonlocal means with a non-local partial differential equation is defined by Gillboa and Osher [8] . The nonlocal operator is based on the image slice similarity which use one patch around pixels to look for another similar patch of the image in detail. It defines as follows:

$$
w(x, y)=\exp \left\{-\frac{G_{\sigma} *(\| f(x+\bullet)-f(y+\bullet) \mid)^{2}}{h^{2}}\right\}
$$

The image $\mathrm{f}(\mathrm{x})$ is defined on the image space $\Omega: \Omega \rightarrow \mathrm{R}, x \in \Omega, y \in \Omega$, in which $G_{\sigma}$ is the Gaussian kennel function. $\sigma$ is the width parameter of the gaussian kernel function, h is the threshold value for similarities between two patch windows. $\mathrm{w}: \Omega \times \Omega \rightarrow \mathrm{R}$ is the edge weight between the points $\mathrm{x}, \mathrm{y}$. In general, $\mathrm{w}(\mathrm{x}, \mathrm{y})$ defines a graph. In this paper, we consider that $\mathrm{w}$ is symmetric which is $\mathrm{w}(\mathrm{x}$, $y)=w(y, x)$. The nonlocal gradient for two points $x$ and $y$ in the image is defined as

$$
\nabla_{N L} u(x, y)=(u(y)-u(x)) \sqrt{w(x, y)}: x, y \in \Omega
$$

The nonlocal gradient is not the general vector but the map of $\Omega \times \Omega \rightarrow R$ in the standard image. The nonlocal vector is $\mathrm{v}(\mathrm{x}, \mathrm{y}): \Omega \times \Omega \rightarrow R$, the inner product is given by

$$
\left\langle v_{1} \bullet v_{2}\right\rangle(x)=\int_{\Omega} v_{1}(x, y) v_{2}(x, y) d x: \Omega \rightarrow R
$$

The module is

$$
|v|(x)=\sqrt{\int_{\Omega}(v(x, y))^{2} d y}: \Omega \rightarrow R
$$

With the inner product, The nonlocal divergence is defined as

$$
\left(\nabla_{N L} \bullet v\right)(x)=\int_{\Omega}(v(x, y)-v(y, x)) \sqrt{w(x, y)} d y
$$

Then the nonlocal laplacian can be defined as

$$
\Delta_{N L} u(x)=\frac{1}{2}\left(\nabla_{N L} \bullet\left(\nabla_{N L} u\right)\right)(x)=\int_{\Omega}(u(y)-u(x)) w(x, y) d y
$$

With the above mentioned definitions, we can write the norm of the nonlocal gradient for a function $\mathrm{u}$ as follows.

$$
\left|\nabla_{N L} u\right|(x)=\sqrt{\int_{\Omega}(u(y)-u(x))^{2} w(x, y) d y}: \Omega \rightarrow R
$$

For the gray image $\mathrm{f}(\mathrm{x})$ of defining on the image space $\Omega: \Omega \rightarrow \mathrm{R}$, the active contour model for the constrained optimization problem is defined in the document [13] .

$$
\operatorname{Min}_{u_{1}, u_{2}, \phi \in\{0,1\}}\left\{E\left(u_{1}, u_{2}, \phi\right)=\alpha_{1} \int_{\Omega}\left(\left(u_{1}-f\right)^{2}+\lambda_{1}\left|\nabla u_{1}\right|^{2}\right) \phi d x+\alpha_{2} \int_{\Omega}\left(\left(u_{2}-f\right)^{2}+\lambda_{2}\left|\nabla u_{2}\right|^{2}\right)(1-\phi) d x+\gamma \int_{\Omega}|\nabla \phi| d x\right\}
$$

It uses binary tag function $\phi(x)= \begin{cases}1, & x \in \Omega_{1}, \Omega=\Omega_{1} \cup \Omega_{2}, \Omega_{1} \cap \Omega_{2}=\phi \text { to define the level set function and } \\ 0, & x \in \Omega_{2}\end{cases}$ express the continuous contour lines of region partition. The items $\left(u_{1}-f\right)^{2}$ and $\left(u_{2}-f\right)^{2}$ mean the proximity between the original image with the approximate image of the smooth regions. The items $\lambda_{1}\left|\nabla u_{1}\right|$ and $\lambda_{2}\left|\nabla u_{2}\right|$ are penalty items for piecewise smooth degree. $u_{1}, u_{2}$ means the piecewise 
smooth image of prospect and background, the first two items are the estimated data of the piecewise smooth images. $\Omega_{1}$ means the prospect of the image, $\Omega_{2}=\Omega \backslash \Omega_{1}$ means the background of the image, $\Gamma$ means the contour line. $\alpha_{1}, \alpha_{2}, \lambda_{1}, \lambda_{2}, \gamma$ are the penalty parameters.

According to the formula (8), the active contour model for color image segmentation is defined as follows.

$$
\underset{u, \phi \in\{0,1\}}{\operatorname{Min}}\left\{E\left(u_{i j}, \phi\right)=\sum_{i=1}^{2} \sum_{j=1}^{3} \alpha_{i} \int_{\Omega}\left(\left(u_{i j}-f\right)^{2}+\lambda_{i}\left|\nabla u_{i j}\right|\right) \chi_{i}(\phi) d x+\sum_{i=1}^{1} \gamma \int_{\Omega}\left|\nabla \phi_{i}\right| d x\right\}
$$

$\chi_{i}(\phi)$ can be defined as follows.

$$
\chi_{i}(\phi)=\phi_{i} \prod_{j=0}^{i-1}\left(1-\phi_{j}\right)\left(\phi_{0} \equiv 0, \phi_{n+1} \equiv 1\right)
$$

The formula (9) is for color image segmentation. The color image can be seen as the three channels of R G B, so $\mathrm{j}=1,2,3$. However this formula can only use for easy color images which do not contain a lot of complicated information especially the textures features.

Base on the above description, we modified the above model (9) for the color image which contains texture characteristics. The corresponding energy function extremum problem is the follows model.

$$
\underset{u, \phi \in\{0,1\}}{\operatorname{Min}}\left\{E\left(u_{i j}, \phi\right)=\sum_{i=1}^{2} \sum_{j=1}^{3} \alpha_{i} \int_{\Omega}\left(\left(u_{i j}-f\right)^{2}+\lambda_{i}\left|\nabla_{N L} u_{i j}\right|^{2}\right) \chi_{i}(\phi) d x+\sum_{i=1}^{1} \gamma \int_{\Omega}\left|\nabla \phi_{i}\right| d x\right\}
$$

$f(x)=\left(f_{1}(x), f_{2}(x), \ldots, f_{n}(x)\right): \Omega \in R^{n}$ is color texture image. The first item $\left(u_{i j}-f\right)^{2}$ means the proximity between the original image with the approximate image of the smooth regions. The item $\lambda_{i}\left|\nabla_{N L} u_{i j}\right|^{2}$ are all the penalty items for piecewise smooth degree. The second item of the two penalty items is nonlocal term which is used to classify the similar texture features. The nonlocal term is based on the image slice similarity. $\alpha, \gamma, \lambda$ are the penalty parameters. The larger $\lambda$ is, the more smooth the piecewise image becomes. The larger the $\gamma$ is, the shorter the segmentation-line becomes. The last item is the same with the formula (9). When $\lambda_{i}$ is zero, the formula (11) is the conventional active contours model. This model can segment not only the conventional images but also the texture images. The answer in detail of the formula (11) is defined as follows. The energy function about $u$ is

$$
\operatorname{Min}_{u_{i j}}\left\{E\left(u_{i j}\right)=\sum_{i=1}^{2} \sum_{j=1}^{3} \alpha_{i} \int_{\Omega}\left(\left(u_{i j}-f\right)^{2}+\lambda_{i}\left|\nabla_{N L} u_{i j}\right|^{2}\right) \chi_{i}(\phi) d x\right\}
$$

The Euler-Lagrange equation solved by the variational method about $\mathrm{u}$ is

$$
\left(u_{i j}-f\right) \chi_{i}(\phi)-\lambda_{i} \nabla_{N L} \bullet\left(\chi_{i}(\phi)\left|\nabla_{N L} u_{i j}\right|\right)=0
$$

Considering the equation (13), we can obtain $u(x)$ as follows

$$
u_{i j}=\frac{f(x) \chi_{i}(\phi)+\lambda_{i} \int_{\Omega}\left(\chi_{i}(\phi(y))+\chi_{i}(\phi(x))\right) u_{i j}(y) w(x, y) d y}{\chi_{i}(\phi)+\lambda_{i} \int_{\Omega}\left(\chi_{i}(\phi(y))+\chi_{i}(\phi(x))\right) w(x, y) d y}
$$

The energy function about $\phi$ is

$$
\underset{\phi_{i}}{\operatorname{Min}}\left\{E\left(\phi_{i}\right)=\sum_{i=1}^{2} \sum_{j=1}^{3} \int_{\Omega}\left(\left(u_{i j}-f\right)^{2}+\lambda_{i}\left|\nabla_{N L} u_{i j}\right|^{2}\right) \chi_{i}(\phi) d x+\sum_{i=1}^{1} \gamma\left|\nabla \phi_{i}\right| d x\right\}
$$

This paper designs to use the split-Bregman iteration method, the instrumental variable $v_{i}$ and Bregman iteration parameter $b_{i}$ are introduced, then the formula (16) is transformed into the iterative optimization format. 


$$
\underset{\phi_{i} \in\{0,1\}}{\operatorname{Min}}\left\{E(\phi, w)=\sum_{i=1}^{2} \sum_{j=1}^{3} \alpha_{i} \int_{\Omega} Q_{i j} \chi_{i}(\phi) d x+\sum_{i=1}^{1} \gamma\left|v_{i}\right| d x+\sum_{i=1}^{1} \frac{\theta}{2} \int_{\Omega}\left(v_{i}-\nabla \phi-b_{i}^{k+1}\right)^{2} d x\right\}
$$

And the constrains is defined by

The form of $Q_{i}(u)$ is

$$
b_{i}^{k+1}=b_{i}^{k}+\nabla_{N L} u_{i}^{k}-v_{i}^{k}, b_{i}^{0}=v_{i}^{0}=0
$$

$$
Q_{i j}(u)=\left(u_{i j}-f\right)^{2}+\lambda_{i}\left|\nabla_{N L} u_{i j}\right|^{2}
$$

The Euler-Lagrange equation about $\phi$ and the approximate generalized soft threshold formula about $v_{i}$ based on the alternating iterative optimization strategy and variational method can be obtained.

$$
\left\{\begin{array}{cc}
\nabla \bullet\left(V_{i}-\nabla \phi-b_{i}^{k+1}\right)+\sum_{i=1}^{2} Q_{i j}(u) \frac{\partial \chi_{i}(\phi)}{\partial \phi}=0 & \text { in } \Omega \\
\left(v_{i}-\nabla \phi_{i}-b_{i}^{k+1}\right) \bullet \vec{n}=0 & \text { on } \Omega
\end{array}\right.
$$

In the equation (19), the form of $\frac{\partial \chi_{i}(\phi)}{\partial \phi_{k}}$ is

$$
\frac{\partial \chi_{i}(\phi)}{\partial \phi_{k}}=\left\{\begin{array}{cc}
\prod_{j=0}^{i-1}\left[1-\phi_{j}\right] & k=i \\
-\prod_{j=0, k \neq i}^{i-1}\left[1-\phi_{j}\right] \phi_{i} & k \neq i
\end{array}\right.
$$

With the equation (20), we can obtain $v_{i}^{k+1}$ as follows.

$$
v_{i}^{k+1}=\operatorname{Max}\left(\left|\nabla \phi_{i}^{k+1}+b_{i}^{k+1}\right|-\frac{\gamma}{\theta}, 0\right) \frac{\nabla \phi_{i}^{k+1}+b_{i}^{k+1}}{\left|\nabla \phi_{i}^{k+1}+b_{i}^{k+1}\right|}
$$

Where

$$
\phi_{i}=\operatorname{Max}\left(\operatorname{Min}\left(\phi_{i}, 1\right), 0\right)
$$

\section{Test results}

In the section, we firstly segment three complex texture images in Fig1. Then we make some comparisons for some real color texture images from the well-know Berkeley Segmentation Dataset [10] in Fig3.
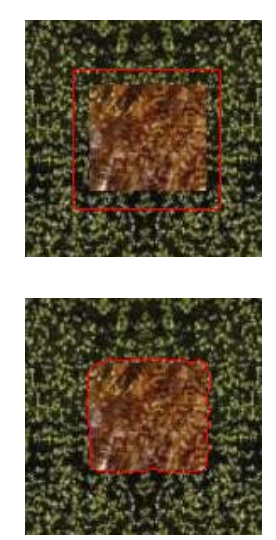

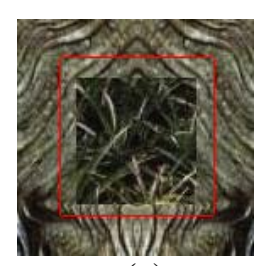

(a)

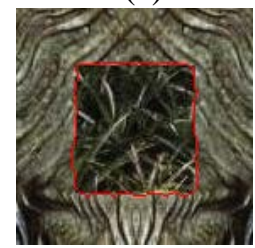

(b)
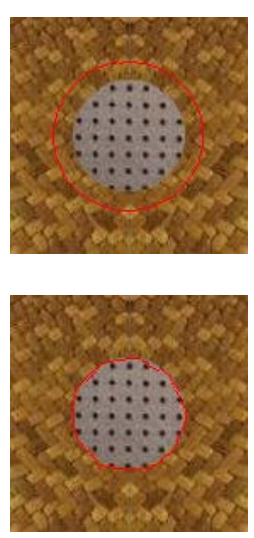

Fig.1. the segmentation of complex texture images: (a) are the initialized images with the initialized contour line. (b) are the segmentation results.

Fig.1 (a) is the origin texture images; Fig.1 (b) is the result of segmentation. The three images' target regions and the background regions have some similarities in texture features. The algorithm gives good results. 

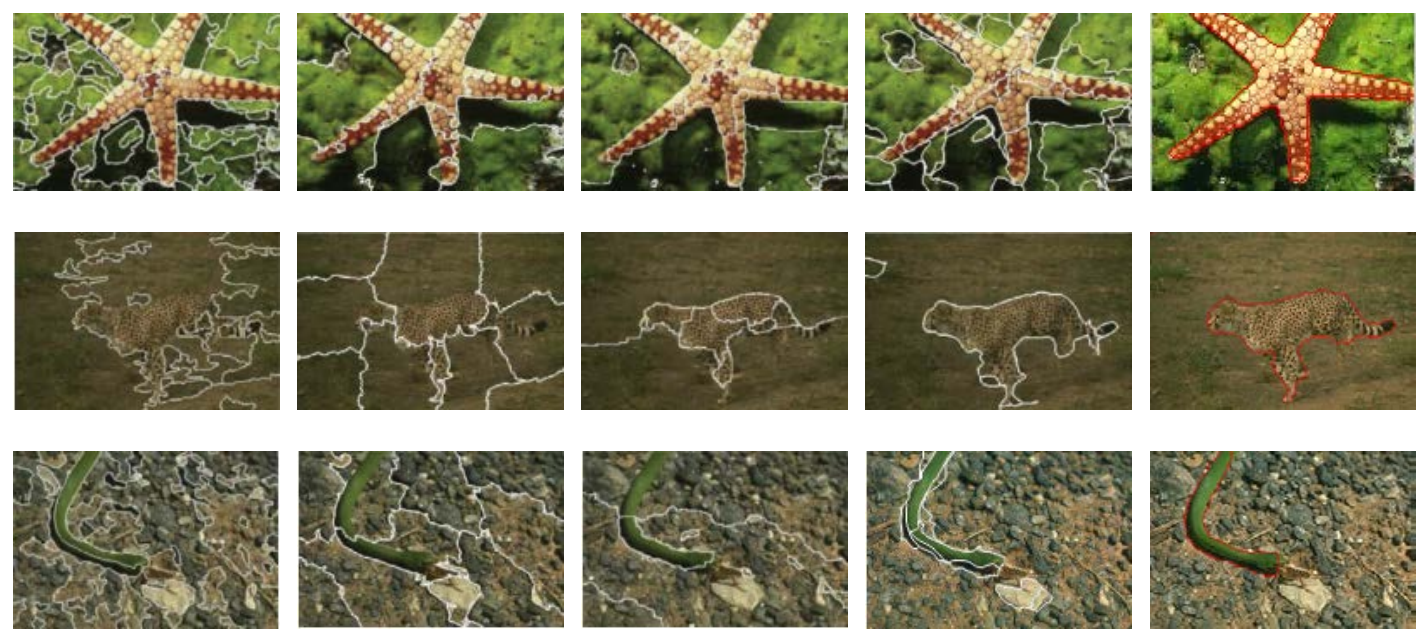

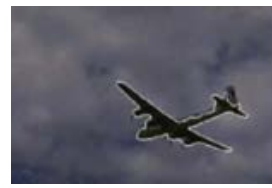

(a)

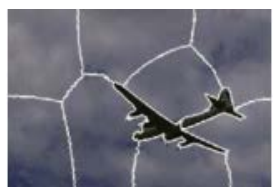

(b)

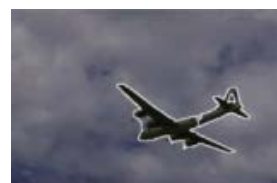

(c)

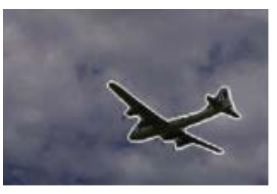

(d)

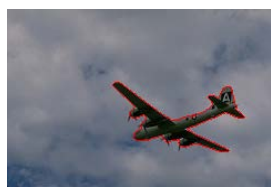

(e)

Fig.2.Comparison results between our method and some other methods in: (a) is the result by JSEG method, (b) is the result by NCut method, (c) is the result by MAP-ML method, (d) is the result by CTM method, (e) is the result by our proposed method.

In order to demonstrate the superiority of our proposed model, the comparison experiments between our method and other methods are shown in Fig.2. The methods contain JSEG algorithm [6], CTM algorithm [11], NCut [12], and the MAP-ML estimations algorithm[13]. We can find that our contour line is fully close to the object region. Our method has better results. For the starfish, all legs of starfish are fully fit with the contour line. And to some degree, it is not affected by the background, especially the white region of the lower right corner. For the leopard, its foot which trample on the grass is segmented well. Meanwhile compared with other methods the tail is also perfectly segmented. It's also not affected by the interference of background color. The snake has no confused with the background which has many stones. Then the airplane have good segmentation result too. The back fuselage section is better segmented particularly than other methods.

\section{Conclusion}

In this paper, by using the relevant concepts of nonlocal operators and the active contour model, we proposed this model based on active contour model with nonlocal Tikhonov regularization for color texture image segmentation. In this model, we design a Split-Bregman algorithm and provide the implementations. Numerical experiments confirm the performance of the proposed model for color texture images segmentation.

\section{Acknowledgement}

This work was supported by National Natural Science Foundation of China (No.61305045 and No.61170106), National "Twelfth Five-Year" development plan of science and technology (No.2013BAI01B03), Qingdao science and technology development project (No. 13-1-4-190-jch). 


\section{References}

[1] D. Lui, C. Scharfenberger, K. Fergani, et al., Enhanced Decoupled Active Contour Using Structural and Textural Variation Energy Functionals. Image Processing, IEEE Transactions on, 2014, 23(2): 855 - 869.

[2] C. Xu, L. Prince, Snakes, Shapes and Gradient Vector Flow. IEEE Transactions on Image Processing 7 (3) (1998) 359-369.

[3] V. Caselles, F. Catte, T. Coll, F. Dibos, A geometric model for active contours in image processing.Num. Math. 66 (1993) 1-31.

[4] M. Kass, A. Witkin, D. Terzopoulos, Snakes:Active Contour Models.International Journal of Computer Vision 1 (1998) 321-331.

[5] V. Caselles, R. Kimmel, G. Sapiro, On geodesic active contours, Int. J. of Computer Vision 22 (1) (1997) 61-79, 1997.

[6] Y. Deng, B. S. Manjunath Unsupercised segmentation of color-texture regions in images and video. IEEE Trans.Pattern Analysis Machine Intelligence,2001,23 (8): 800-810.

[7] T. Goldstein, X. Bresson, S. Osher, Geometric Applications of the Split Bregman Method. Segmentation and Surface Reconstruction, CAM Report 09-06, 2009.

[8] G. Gilboa and S. Osher, “Nonlocal Operators with Applications to Image Processing,” SIAM Multiscale Modeling Simulation, Vol. 7, No. 3, 2008, pp. 1005-1028.

[9] X. Bresson, S. Esedoglu, P. Vandergheynst, JP. Thiran, S. Osher, Fast global minimization of the active contour/snake model. Journal of Mathematical Imaging and Vision, 2007, 28 (2): $151-167$.

[10]D. Martin, C. Fowlkes, D. Tal, and J. Malik, A database of human segmented natural images and its application to evaluating segmentation algorithms and measuring ecological statistics. in Proceedings of the 8th International Conference on Computer Vision, pp. 416-423, July 2001.

[11]A. Y. Yang, J. Wright, Y. Ma and S. S. Sastry, Unsupervised segmentation of natural images via lossy data compression. Computer Vision and Image Understanding, vol. 110, no. 2, pp. 212-225, 2008.

[12]J. Shi and J. Malik, Normalized cuts and image segmentation. IEEE Transactions on Pattern Analysis and Machine Intelligence, vol. 22, no. 8, pp. 888-905, 2000.

[13] S. Chen, L. Cao, Y. Wang, J. Liu, and X. Tang, Image segmentation by MAP-ML estimations. IEEE Transactions on Image Processing, vol. 19, no. 9, pp. 2254-2264, 2010. 